\title{
A note on the Laplace operator for holomorphic functions on complex Lie groups
}

\author{
Alexandru Ionescu
}

\begin{abstract}
In this note we obtain the local expression of the Laplace operator acting on holomorphic functions defined on a complex Lie group. Also, some applications to the theory of holomorphic last multipliers are given.
\end{abstract}

Mathematics Subject Classification (2010): 22E10, 32M05, 53B20, 53B21, 53C56, $34 \mathrm{~A} 26$.

Keywords: Complex Lie group, holomorphic function, Laplace operator, last multiplier.

\section{Preliminaries on complex Lie groups}

Let $G$ be a complex Lie group of dimension $n$. Its Lie algebra, $\mathfrak{g}$, has as underlying vector space the holomorphic tangent space $T_{e}^{1,0} G$ at the identity $e \in G$. As known, an element $A \in T_{e}^{1,0} G$ determines a unique left invariant vector field which takes the value $A$ at $e$; moreover, these vector fields are the elements of $\mathfrak{g}$.

Following the ideas from [1], let $\left\{E_{\alpha}\right\}, \alpha=1, \ldots, n$, be a base of the Lie algebra $\mathfrak{g}$ and $\chi^{\alpha}, \alpha=1, \ldots, n$ the dual base for the 1-forms of Maurer-Cartan, that is, $\chi^{\alpha}\left(E_{\beta}\right)=\delta_{\beta}^{\alpha},(\alpha, \beta=1, \ldots, n)$. It is known ([11], Lemma 1.6) that $E_{\alpha}$ are holomorphic vector fields (as they are left-invariant) and also $\chi^{\alpha}$ are holomorphic left-invariant 1-forms.

A differential form $\eta$ is said to be left-invariant if it is invariant by every left translation $L_{a},(a \in G)$, that is, if $L_{a}^{*} \eta=\eta$ for every $a \in G$, where $L_{a}^{*}$ is the holomorphic cotangent map of $L_{a}$. It follows that any left invariant form must be holomorphic. For an element $U \in \mathfrak{g}$ and an element $\eta$ in the dual space $\mathfrak{g}^{*}, \eta(U)$ is constant on $G$. Since

$$
\partial \eta(U, V)=U \eta(V)-V \eta(U)-\eta([U, V]),
$$

where $d=\partial+\bar{\partial}$ is the usual decomposition of the exterior derivative, one obtains

$$
\partial \eta(U, V)=-\eta([U, V]),
$$


where $U, V$ are elements of $\mathfrak{g}$ and $\eta$ is any element of the dual space. By setting

$$
\left[E_{\beta}, E_{\gamma}\right]=C_{\beta \gamma}^{\alpha} E_{\alpha},
$$

the relation (1.1) yields

$$
\partial \chi^{\alpha}=-\frac{1}{2} C_{\beta \gamma}^{\alpha} \chi^{\beta} \wedge \chi^{\gamma}
$$

The complex constants $C_{\beta \gamma}^{\alpha}$ are called the constants of structure of $\mathfrak{g}$ with respect to the holomorphic base $\left\{E_{1}, \ldots, E_{n}\right\}$. These constants are not arbitrary since they must satisfy the relations

$$
\left[E_{\alpha}, E_{\beta}\right]+\left[E_{\beta}, E_{\alpha}\right]=0
$$

and

$$
\left[E_{\alpha},\left[E_{\beta}, E_{\gamma}\right]\right]+\left[E_{\beta},\left[E_{\gamma}, E_{\alpha}\right]\right]+\left[E_{\gamma},\left[E_{\alpha}, E_{\beta}\right]\right]=0
$$

for all $\alpha, \beta, \gamma=1, \ldots, n$, that is

$$
C_{\beta \gamma}^{\alpha}+C_{\gamma \beta}^{\alpha}=0
$$

and

$$
C_{\alpha \beta}^{\rho} C_{\gamma \rho}^{\delta}+C_{\beta \gamma}^{\rho} C_{\alpha \rho}^{\delta}+C_{\gamma \alpha}^{\rho} C_{\beta \rho}^{\delta}=0 .
$$

Equations (1.3) are called the holomorphic Maurer-Cartan equations.

Equation (1.2) indicates that the structure constants are the components of a holomorphic tensor on $T_{e}^{1,0} G$ of type $(1,2)$. A new holomorphic tensor on $T_{e}^{1,0} G$ can be defined by setting

$$
C_{\alpha \beta}=C_{\alpha \sigma}^{\rho} C_{\rho \beta}^{\sigma}
$$

with respect to the holomorphic left invariant base $\left\{E_{\alpha}\right\}(\alpha=1, \ldots, n)$ of $\mathfrak{g}$. It is easily verified that this holomorphic tensor is symmetric. Also, it can be shown that a necessary and sufficient condition for the complex Lie group $G$ to be semi-simple is that the complex matrix $\left(C_{\alpha \beta}\right)_{n \times n}$ is invertible.

The holomorphic tensor defined by the equations (1.8) can now be used to raise and lower indices and, for this purpose, the inverse matrix $\left(C^{\alpha \beta}\right)_{n \times n}$ will be considered.

In terms of a system of local complex coordinates $\left(u^{1}, \ldots, u^{n}\right)$ on $G$, the holomorphic vector fields $E_{\alpha}, \alpha=1, \ldots, n$, can be expressed as $E_{\alpha}=\chi_{\alpha}^{i} \frac{\partial}{\partial u^{i}}$. Since $G$ is complex parallelizable (see [14]), the $n \times n$ matrix $\left(\chi_{\alpha}^{i}\right)$ has rank $n$ and so, by setting

$$
g^{i j}=\chi_{\alpha}^{i} \chi_{\beta}^{j} C^{\alpha \beta},
$$

a positive definite and symmetric matrix $\left(g^{i j}\right)_{n \times n}$ is obtained. Hence, a holomorphic Riemannian metric $g$ on $G$ can be defined by means of the complex quadratic form

$$
d s^{2}=g_{j k} d u^{j} \otimes d u^{k}
$$

where $\left(g_{j k}\right)_{n \times n}$ denotes the matrix inverse to $\left(g^{j k}\right)_{n \times n}$, that is, $g_{j k}=C_{\beta \gamma} \chi_{j}^{\beta} \chi_{k}^{\gamma}$.

Moreover, the holomorphic metric tensor $g$ can be also used to raise and lower indices in the usual manner, and this holomorphic metric is completely determined by the complex Lie group $G$. 
In the following, we define $n$ holomorphic covariant vector fields $\chi^{\alpha}(\alpha=$ $1, \ldots, n)$ on $G$, with local components $\chi_{i}^{\alpha}(i=1, \ldots, n)$ given by

$$
\chi_{i}^{\alpha}=C^{\alpha \beta} \chi_{\beta}^{j} g_{i j}
$$

It easily follows that

$$
\chi_{\alpha}^{i} \chi_{j}^{\alpha}=\delta_{j}^{i} \quad \text { and } \quad \chi_{\alpha}^{i} \chi_{i}^{\beta}=\delta_{\beta}^{\alpha} .
$$

Also, we consider the set of $n^{2}$ linear holomorphic 1-forms $\omega_{j}^{i}=\Gamma_{j k}^{i} d u^{k}$ defined locally by setting

$$
\Gamma_{j k}^{i}=\chi_{\alpha}^{i} \frac{\partial \chi_{j}^{\alpha}}{\partial u^{k}}
$$

By virtue of the equations (1.12), the holomorphic coefficients $\Gamma_{j k}^{i}$ can also be expressed as

$$
\Gamma_{j k}^{i}=-\chi_{j}^{\alpha} \frac{\partial \chi_{\alpha}^{i}}{\partial u^{k}}
$$

and they represent the local coefficients of a left invariant holomorphic connection $\nabla$ on $G$, that is, $\nabla$ is absolutely parallel with respect to every left-invariant holomorphic vector field $U=U^{\alpha} E_{\alpha} \in \mathfrak{g}$.

It is easily verified that in the overlap $U \cap U^{\prime}$ of two local charts, the holomorphic 1 -forms $\omega_{j}^{i}$ change by the rule

$$
\frac{\partial u^{\prime k}}{\partial u^{j}} \omega_{k}^{\prime i}=\frac{\partial u^{i}}{\partial u^{k}} \omega_{j}^{k}-\frac{\partial^{2} u^{i}}{\partial u^{l} \partial u^{j}} d u^{l} .
$$

The next natural step is to consider the torsion of this connection. As in the case of real Lie groups (see $[1,13]$ ), the holomorphic torsion tensor will be written as

$$
T_{j k}^{i}=\frac{1}{2} \chi_{\alpha}^{i}\left(\frac{\partial \chi_{j}^{\alpha}}{\partial u^{k}}-\frac{\partial \chi_{k}^{\alpha}}{\partial u^{j}}\right) .
$$

Since the equations (1.2) can be expressed in terms of the local coordinates $\left(u^{i}\right)$ in the form

$$
\chi_{\beta}^{r} \frac{\partial \chi_{\gamma}^{i}}{\partial u^{r}}-\chi_{\gamma}^{r} \frac{\partial \chi_{\beta}^{i}}{\partial u^{r}}=C_{\beta \gamma}^{\alpha} \chi_{\alpha}^{i}
$$

by using the holomorphic Maurer-Cartan equations (1.3) it easily follows that

$$
T_{j k}^{i}=\frac{1}{2} C_{\beta \gamma}^{\alpha} \chi_{\alpha}^{i} \chi_{j}^{\beta} \chi_{k}^{\gamma}
$$

Also, if we consider the local coefficients of the holomorphic Levi-Civita connection $\stackrel{\circ}{\nabla}$ with respect to the holomorphic metric $g=d s^{2}$ from (1.10) on $G$, they can be expressed as

$$
\stackrel{\circ}{\Gamma}_{j k}^{i}=\frac{1}{2} \chi_{\alpha}^{i}\left(\frac{\partial \chi_{j}^{\alpha}}{\partial u^{k}}+\frac{\partial \chi_{k}^{\alpha}}{\partial u^{j}}\right),
$$

from which follows that

$$
\Gamma_{j k}^{i}=\stackrel{\circ}{\Gamma} \underset{j k}{i}+T_{j k}^{i}
$$

We have

Lemma 1.1. The elements of the Lie algebra $\mathfrak{g}$ of $G$ define holomorphic translations in $G$. 
Proof. It follows in a similar manner to the case of real Lie groups, see [1].

\section{Laplace operators for holomorphic functions on $G$}

In this section, we introduce the Laplace operator acting on holomorphic functions on the complex Lie group $G$, depending on the given holomorphic metric tensor on $G$.

Denote by $\omega=\chi^{1} \wedge \cdots \wedge \chi^{n}$, where $\chi^{i}, i=1, \ldots, n$ are the elements of the base of holomorphic 1-forms defined in Section 1. Then $\omega$ is a nowhere vanishing holomorphic left-invariant $n$-form, called the holomorphic volume element, and it can be used to define the divergence of a holomorphic vector field $U=U^{\alpha} E_{\alpha}$ by setting

$$
\operatorname{div}(U) \omega=\partial\left(i_{U} \omega\right)
$$

Note that the divergence can also be defined by means of the Lie derivative $L_{U}$ with respect with a left invariant holomorphic vector field $U$ :

$$
\operatorname{div}(U) \omega=L_{U} \omega
$$

where

$$
L_{U} \eta=\left.\frac{d}{d t}\right|_{t=0}\left(\varphi_{U}^{t}\right)^{*} \eta
$$

for an arbitrary holomorphic tensor $\eta$. The equivalence between definitions (2.1) and (2.2) is due to Cartan's formula $L_{U} \eta=\partial\left(i_{U} \eta\right)+i_{U} \partial \eta$ for $\eta=\omega$. The first definition is more convenient for computations, though. Another property of the divergence is

$$
\operatorname{div}(f U)=U f+f \operatorname{div} U
$$

for a holomorphic vector field $U$ and a holomorphic function $f$ defined on $G$.

Also, for a given holomorphic vector field $U=U^{\alpha} E_{\alpha}$ on $G$, we have

$$
\operatorname{div} U=E_{\alpha}\left(U^{\alpha}\right) \text {. }
$$

Let $G$ be a semi-simple complex Lie group with the holomorphic Riemannian metric

$$
g=g_{i j} d u^{i} \otimes d u^{j}, \quad g_{i j}=C_{\alpha \beta} \chi_{i}^{\alpha} \chi_{j}^{\beta} .
$$

A simple computation gives $g\left(E_{\alpha}, E_{\beta}\right)=C_{\alpha \beta}$ and the holomorphic metric tensor $g$ will now be used to define the gradient of a holomorphic function $f$ on $G$. If $\operatorname{grad} f=$ $V^{\beta} E_{\beta}$ is a holomorphic vector field defined in a local chart, then the classical definition

$$
g(U, \operatorname{grad} f)=U f
$$

for $U=U^{\alpha} E_{\alpha}$ yields $V^{\beta}=C^{\beta \alpha}\left(E_{\alpha} f\right)$, hence

$$
\operatorname{grad} f=C^{\beta \alpha}\left(E_{\alpha} f\right) E_{\beta} .
$$

A Laplace operator for holomorphic functions on $G$ can now be introduced by

$$
\Delta f=(\operatorname{div} \circ \operatorname{grad}) f=C^{\beta \alpha} E_{\beta}\left(E_{\alpha} f\right) .
$$


In local coordinates, this reads

But

$$
\begin{aligned}
\Delta f & =C^{\beta \alpha} \chi_{\beta}^{i} \frac{\partial}{\partial u^{i}}\left(\chi_{\alpha}^{j} \frac{\partial f}{\partial u^{j}}\right) \\
& =C^{\beta \alpha} \chi_{\beta}^{i} \frac{\partial \chi_{\alpha}^{j}}{\partial u^{i}} \frac{\partial f}{\partial u^{j}}+C^{\beta \alpha} \chi_{\beta}^{i} \chi_{\alpha}^{j} \frac{\partial^{2} f}{\partial u^{i} \partial u^{j}} .
\end{aligned}
$$

$$
\chi_{\beta}^{i} \frac{\partial \chi_{\alpha}^{j}}{\partial u^{i}}=-\chi_{\beta}^{i} \chi_{\alpha}^{k} \Gamma_{k i}^{j}
$$

where $\Gamma_{j k}^{i}=-\chi_{j}^{\beta} \frac{\partial \chi_{\beta}^{i}}{\partial u^{k}}$ are the coefficients of the holomorphic connection written in the form (1.14), such that

$$
\Delta f=g^{i j}\left(\frac{\partial^{2} f}{\partial u^{i} \partial u^{j}}-\Gamma_{j i}^{k} \frac{\partial f}{\partial u^{k}}\right) .
$$

Since

$$
\frac{\partial^{2} f}{\partial u^{i} \partial u^{j}}-\Gamma_{j i}^{k} \frac{\partial f}{\partial u^{k}}=\nabla_{i} \nabla_{j} f,
$$

where $\nabla_{k}$ is the covariant derivative with respect to the left invariant holomorphic connection $\nabla$ defined in the previous section, this leads to the following formula for the Laplace operator of holomorphic functions on $G$ :

$$
\Delta f=g^{i j} \nabla_{i} \nabla_{j} f .
$$

Remark 2.1. If $G$ is not semi-simple then a holomorphic Riemannian metric on $G$ can be defined by setting

$$
h=h_{i j} d u^{i} \otimes d u^{j}, \quad h_{i j}=\delta_{\alpha \beta} \chi_{i}^{\alpha} \chi_{j}^{\beta},
$$

and similar computations as above lead to the following local expression of the Laplacian:

$$
\Delta f=E_{\alpha}^{2} f=h^{i j} \nabla_{i} \nabla_{j} f .
$$

Let us compute the local expression of the Laplacian in two particular cases.

Example 2.2. Consider the standard 4-dimensional complex manifold $\mathbb{C}^{4}$ with the holomorphic coordinates $\left(z^{1}, z^{2}, z^{3}, z^{4}\right)$ and the following multiplication rule:

$$
\begin{aligned}
\left(z^{1}, z^{2}, z^{3}, z^{4}\right) & \cdot\left(w^{1}, w^{2}, w^{3}, w^{4}\right)= \\
= & \left(z^{1} e^{\lambda w^{3}}+w^{1}, z^{2} e^{-\lambda w^{3}}+w^{2}, z^{3}+w^{3}, z^{4}+w^{4}-\lambda z^{1} w^{2} e^{\lambda w^{3}}\right)
\end{aligned}
$$

where $\lambda$ is a nonzero complex parameter. The above multiplication rule endows $\mathbb{C}^{4}$ with a non-abelian complex Lie structure. For $\lambda=0$, we obtain the usual abelian Lie group $\mathbb{C}^{4}$, therefore we will consider here $\lambda \neq 0$. We denote by $G$ the non-abelian complex Lie group $\mathbb{C}^{4}$ endowed with the multiplication rule (2.11).

It is easy to see that the following left-invariant holomorphic vector fields given by

$$
Z_{1}=\frac{\partial}{\partial z^{1}}, \quad Z_{2}=\frac{\partial}{\partial z^{2}}-\lambda z^{1} \frac{\partial}{\partial z^{4}}, \quad Z_{3}=\lambda z^{1} \frac{\partial}{\partial z^{1}}-\lambda z^{2} \frac{\partial}{\partial z^{2}}+\frac{\partial}{\partial z^{3}}, \quad Z_{4}=\frac{\partial}{\partial z^{4}}
$$


form a basis of the holomorphic Lie algebra $\mathfrak{g}$ of $G$. If we compute the Lie brackets of these holomorphic vector fields, we obtain

$$
\begin{aligned}
{\left[Z_{1}, Z_{2}\right]=-\lambda Z_{4}, \quad\left[Z_{1}, Z_{3}\right] } & =\lambda Z_{1}, \quad\left[Z_{2}, Z_{3}\right]=-\lambda Z_{2}, \\
{\left[Z_{1}, Z_{4}\right]=\left[Z_{2}, Z_{4}\right] } & =\left[Z_{3}, Z_{4}\right]=0,
\end{aligned}
$$

therefore, the components of the Lie brackets are constant. Hence, they are the structure constants of $\mathfrak{g}$ with respect to the basis $\left\{Z_{1}, Z_{2}, Z_{3}, Z_{4}\right\}$. We have $C_{\alpha \beta}^{\gamma}=0$, $\alpha, \beta, \gamma=\overline{1,4}$ with the following exceptions:

$$
C_{12}^{4}=-\lambda, \quad C_{13}^{1}=\lambda, \quad C_{23}^{2}=-\lambda .
$$

The tensor field introduced by (1.8) will consequently vanish, i.e., $C_{\alpha \beta}=0$ for all $\alpha, \beta=\overline{1,4}$, which means that $G$ is not semi-simple. Then, according to $(2.10)$, the Laplace operator $\Delta$ acting on holomorphic functions $f \in \operatorname{Hol}\left(\mathbb{C}^{4}\right)$ is

$$
\Delta f=\sum_{\alpha} Z_{\alpha}^{2} f=Z_{1}^{2} f+Z_{2}^{2} f+Z_{3}^{2} f+Z_{4}^{2} f .
$$

Now, a basic computation using (2.12) gives

$$
\begin{aligned}
\Delta f & =\left(1+\lambda^{2}\left(z^{1}\right)^{2}\right) \frac{\partial^{2} f}{\partial\left(z^{1}\right)^{2}}+\left(1+\lambda^{2}\left(z^{2}\right)^{2}\right) \frac{\partial^{2} f}{\partial\left(z^{2}\right)^{2}}+\frac{\partial^{2} f}{\partial\left(z^{3}\right)^{2}} \\
& +\left(1+\lambda^{2}\left(z^{1}\right)^{2}\right) \frac{\partial^{2} f}{\partial\left(z^{4}\right)^{2}}-2 \lambda^{2} z^{1} z^{2} \frac{\partial^{2} f}{\partial z^{1} \partial z^{2}}+2 \lambda z^{1} \frac{\partial^{2} f}{\partial z^{1} \partial z^{3}} \\
& -2 \lambda z^{2} \frac{\partial^{2} f}{\partial z^{2} \partial z^{3}}-2 \lambda z^{1} \frac{\partial^{2} f}{\partial z^{2} \partial z^{4}}+\lambda^{2} z^{1} \frac{\partial f}{\partial z^{1}}+\lambda^{2} z^{2} \frac{\partial f}{\partial z^{2}}
\end{aligned}
$$

Example 2.3. Let $G=\mathbb{C}^{*} \times \mathbb{C}$ with the multiplication

$$
\left(z^{1}, z^{2}\right) \circ\left(w^{1}, w^{2}\right)=\left(z^{1} w^{1}, \frac{1}{2} w z^{1} w^{2}+z^{2}\left(w^{1}\right)^{2}\right)
$$

and consider the vector fields $Z_{1}=z^{1} \frac{\partial}{\partial z^{1}}+2 z^{2} \frac{\partial}{\partial z^{2}}, Z_{2}=z^{1} \frac{\partial}{\partial z^{2}}$. Then, $(G, \circ)$ is a complex Lie group with the holomorphic Lie algebra $\mathfrak{g}=\operatorname{span}\left\{Z_{1}, Z_{2}\right\}$. Moreover, $G$ is not semi-simple, as it can be easily shown by computating the tensor $C_{\alpha \beta}$, as in the previous example. We therefore have $\Delta f=Z_{1}^{2} f+Z_{2}^{2} f$, which yields the Laplacian in the form

$$
\begin{aligned}
\Delta f & =\left(z^{1}\right)^{2} \frac{\partial^{2} f}{\partial\left(z^{1}\right)^{2}}+\left(\left(z^{1}\right)^{2}+4\left(z^{2}\right)^{2}\right) \frac{\partial^{2} f}{\partial\left(z^{2}\right)^{2}} \\
& +4 z^{1} z^{2} \frac{\partial^{2} f}{\partial z^{1} \partial z^{2}}+z^{1} \frac{\partial f}{\partial z^{1}}+4 z^{2} \frac{\partial f}{\partial z^{2}} .
\end{aligned}
$$

We will use this example later for illustrating another property of the Laplace operator.

A straightforward computation gives an interesting property of the Laplacian introduced above in the general case. 
Proposition 2.4. The following identity holds:

$$
\left[\Delta, E_{\alpha}\right]=2\left(h^{i j} \chi_{\alpha}^{k}-h^{i k} \chi_{\alpha}^{j}\right) \Gamma_{j k}^{l} \frac{\partial^{2}}{\partial u^{i} \partial u^{l}},
$$

where $h^{i j}=\delta^{\alpha \beta} \chi_{\alpha}^{i} \chi_{\beta}^{j}$ and $\Gamma_{j k}^{l}$ are the local coefficients of the holomorphic connection $\nabla$.

Let us check the result in the case of the Lie group $G=\mathbb{C}^{*} \times \mathbb{C}$ from Example 2.3.

Example 2.5. First, we compute

$$
\begin{aligned}
& {\left[\Delta, Z_{1}\right] f=2\left(z^{1}\right)^{2} \frac{\partial^{2} f}{\partial\left(z^{2}\right)^{2}}} \\
& {\left[\Delta, Z_{2}\right] f=-2\left(z^{1}\right)^{2} \frac{\partial^{2} f}{\partial z^{1} \partial z^{2}}-4 z^{1} z^{2} \frac{\partial^{2} f}{\partial\left(z^{2}\right)^{2}} .}
\end{aligned}
$$

Then, from $Z_{\alpha}=\chi_{\alpha}^{i} \frac{\partial}{\partial z^{i}}$ we get

$$
\chi_{1}^{1}=z^{1}, \quad \chi_{1}^{2}=2 z^{2}, \quad \chi_{2}^{1}=0, \quad \chi_{2}^{2}=z^{1},
$$

such that, using (1.13), we can compute the coefficients $\Gamma_{j k}^{l}$ :

$$
\begin{gathered}
\Gamma_{11}^{1}=-\frac{1}{z^{1}}, \quad \Gamma_{12}^{1}=\Gamma_{21}^{1}=\Gamma_{22}^{1}=0, \\
\Gamma_{11}^{2}=\frac{2 z^{2}}{\left(z^{1}\right)^{2}}, \quad \Gamma_{12}^{2}=-\frac{2}{z^{1}}, \quad \Gamma_{21}^{2}=-\frac{1}{z^{1}}, \quad \Gamma_{22}^{2}=0 .
\end{gathered}
$$

We also need $h^{i j}=\delta^{\alpha \beta} \chi_{\alpha}^{i} \chi_{\beta}^{j}$, that is,

$$
h^{11}=\left(z^{1}\right)^{2}, \quad h^{12}=h^{21}=2 z^{1} z^{2}, \quad h^{22}=\left(z^{1}\right)^{2}+4\left(z^{2}\right)^{2} .
$$

Hence, replacing the nonzero terms in the left-hand side of the first identity (2.14) and doing a straightforward computation yields

$$
\begin{aligned}
{\left[\Delta, Z_{1}\right] f } & =2\left(h^{i j} \chi_{1}^{k}-h^{i k} \chi_{1}^{j}\right) \Gamma_{j k}^{l} \frac{\partial^{2}}{\partial z^{i} \partial z^{l}} \\
& =2\left[\left(h^{11} \chi_{1}^{2}-h^{12} \chi_{1}^{1}\right) \Gamma_{12}^{2}+\left(h^{12} \chi_{1}^{1}-h^{11} \chi_{1}^{2}\right) \Gamma_{21}^{2}\right] \frac{\partial^{2} f}{\partial z^{1} \partial z^{2}} \\
& +2\left[\left(h^{21} \chi_{1}^{2}-h^{22} \chi_{1}^{1}\right) \Gamma_{12}^{2}+\left(h^{22} \chi_{1}^{1}-h^{21} \chi_{1}^{2}\right) \Gamma_{21}^{2}\right] \frac{\partial^{2} f}{\partial\left(z^{2}\right)^{2}} \\
& =2\left(z^{1}\right)^{2} \frac{\partial^{2} f}{\partial\left(z^{2}\right)^{2}},
\end{aligned}
$$

since the first term vanishes. The second identity from (2.14) follows analogously.

We shall also illustrate the property from Proposition 2.4 in the case of the complex Lie group $G L(n, \mathbb{C})$.

Example 2.6. As $\operatorname{dim}(G L(n, \mathbb{C}))=n^{2}$, all the indices from the general case will be replaced by pairs of indices, for instance $\alpha$ becomes $\left(\begin{array}{c}\alpha \\ \beta\end{array}\right), i$ becomes $\left(\begin{array}{c}i \\ m\end{array}\right)$, etc. As a 
convention, these pairs will be rewritten in a manner that should be clear from the text below.

First, let $u \in G L(n, \mathbb{C})$ be a complex matrix with elements $\left\{A_{i}^{\alpha}\right\}$, such that a left-invariant holomorphic vector field will be denoted by

$$
E_{\alpha}^{\beta}:=E_{\left(\begin{array}{c}
\alpha \\
\beta
\end{array}\right)}=\chi_{\left(\begin{array}{c}
\alpha \\
\beta
\end{array}\right)}^{\left(\begin{array}{c}
i \\
m
\end{array}\right)} \frac{\partial}{\partial u^{\left(\begin{array}{c}
i \\
m
\end{array}\right)}}=: \chi_{\alpha m}^{i \beta} \frac{\partial}{\partial u_{m}^{i}},
$$

where $\chi_{\alpha m}^{i \beta} \frac{\partial}{\partial u_{m}^{i}}=\delta_{\alpha}^{i} A_{m}^{\beta}$ (see [7] for more details). The holomorphic Riemannian metric is

$$
h^{\left(\begin{array}{c}
i \\
m
\end{array}\right)\left(\begin{array}{c}
j \\
n
\end{array}\right)}=: h_{m n}^{i j}=\delta^{\alpha \beta} \delta_{\nu \mu} \chi_{\alpha m}^{i \nu} \chi_{\beta n}^{j \mu}
$$

(the group $G L(n, \mathbb{C})$ is not semi-simple). The local coefficients of the holomorphic connection defined in Section 1 are

$$
\Gamma_{\left(\begin{array}{l}
j \\
n
\end{array}\right)\left(\begin{array}{l}
\left(\begin{array}{l}
l \\
q
\end{array}\right) \\
p
\end{array}\right)}=: \Gamma_{j k q}^{l n p}=\chi_{j \tau}^{\varepsilon n} \frac{\partial \chi_{\varepsilon q}^{l \tau}}{\partial u_{p}^{k}} .
$$

These yield

$$
\begin{aligned}
{\left[\Delta, E_{\gamma}\right] f=} & 2\left(h_{m n}^{i j} \chi_{\gamma p}^{k \sigma}-h_{m p}^{i k} \chi_{\gamma n}^{j \sigma}\right) \Gamma_{j k q}^{l n p} \frac{\partial^{2} f}{\partial u_{m}^{i} \partial u_{q}^{l}} \\
= & -2\left(\delta^{\alpha \beta} \delta_{\nu \mu} \chi_{\alpha m}^{i \nu} \chi_{\beta n}^{j \mu} \chi_{\gamma p}^{k \sigma}-\delta^{\alpha \beta} \delta_{\nu \mu} \chi_{\alpha m}^{i \nu} \chi_{\beta p}^{k \mu} \chi_{\gamma n}^{j \sigma}\right) \chi_{j \tau}^{\varepsilon n} \frac{\partial \chi_{\varepsilon q}^{l \tau}}{\partial u_{p}^{k}} \frac{\partial^{2} f}{\partial u_{m}^{i} \partial u_{q}^{l}} \\
= & -2\left(\delta^{\alpha \beta} \delta_{\nu \mu} \delta_{\alpha}^{i} A_{m}^{\nu} \delta_{\beta}^{j} A_{n}^{\mu} \delta_{\gamma}^{k} A_{p}^{\sigma} \delta_{j}^{\varepsilon} A_{\tau}^{n} \delta_{\varepsilon}^{l} \frac{\partial A_{q}^{\tau}}{\partial u_{p}^{k}}\right. \\
& \left.-\delta^{\alpha \beta} \delta_{\nu \mu} \delta_{\alpha}^{i} A_{m}^{\nu} \delta_{\beta}^{k} A_{p}^{\mu} \delta_{\gamma}^{j} A_{n}^{\sigma} \delta_{j}^{\varepsilon} A_{\tau}^{n} \delta_{\varepsilon}^{l} \frac{\partial A_{q}^{\tau}}{\partial u_{p}^{k}}\right) \frac{\partial^{2} f}{\partial u_{m}^{i} \partial u_{q}^{l}} \\
= & 2\left(\delta_{\nu \mu} A_{m}^{\nu} A_{p}^{\sigma} \frac{\partial A_{q}^{\mu}}{\partial u_{p}^{k}}-\delta_{\nu \mu} A_{m}^{\nu} A_{p}^{\mu} \frac{\partial A_{q}^{\sigma}}{\partial u_{p}^{k}}\right) \frac{\partial^{2} f}{\partial u_{m}^{i} \partial u_{q}^{l}} .
\end{aligned}
$$

Remark 2.7. Denoting by $\stackrel{\circ}{\nabla}$ the covariant derivative with respect to the Levi-Civita connection, the substitution of (1.19) in (2.7) yields

$$
\begin{aligned}
\Delta f & =g^{i j}\left(\frac{\partial^{2} f}{\partial u^{i} \partial u^{j}}-\stackrel{\circ}{\Gamma}_{j i}^{k} \frac{\partial f}{\partial u^{k}}\right)-T_{j i}^{k} \frac{\partial f}{\partial u^{k}} \\
& =g^{i j} \stackrel{\circ}{\nabla}_{i}^{\circ} \stackrel{\circ}{\nabla}_{j} f-T_{j i}^{k} \frac{\partial f}{\partial u^{k}},
\end{aligned}
$$

such that a harmonic holomorphic function $f$ on $G$ must satisfy the identity

$$
T_{j i}^{k} \frac{\partial f}{\partial u^{k}}=g^{i j} \stackrel{\circ}{\nabla}_{i} \stackrel{\circ}{\nabla}_{j} f .
$$

Note that $T_{j k}^{i}$ is the holomorphic torsion tensor of the holomorphic connection from (1.13). 


\section{Holomorphic last multipliers for holomorphic vector fields on $G$}

The holomorphic volume element $\omega$ on $G$ defined in Section 2 will now be used to introduce the notion of holomorphic last multipliers. The computations are similar to the case of smooth manifolds, $[2,3,4,5]$, or complex manifolds [6]. More precisely, consider a holomorphic vector field of the form $U=U^{i} \frac{\partial}{\partial u^{i}}, \theta=i_{U} \omega$ and let

$$
\frac{d u^{i}}{d t}=U^{i}\left(u^{1}(t), \ldots, u^{n}(t)\right), 1 \leq i \leq n, t \in \mathbb{R}
$$

be a complex ODE system on $G$ defined by the holomorphic vector field $U$. The classical definition of a last multiplier function for a vector field on smooth manifolds, $[2,3]$, can now be applied to the case of the complex Lie group $G$.

Definition 3.1. A holomorphic function $\mu$ on $G$ is called a holomorphic last multiplier of the complex ODE system generated by $U$ (or holomorphic last multiplier for $U$ ) if

$$
\partial(\mu \theta):=\partial \mu \wedge \theta+\mu \cdot \partial \theta=0 .
$$

Note that for every holomorphic function $\mu$ on $G, \partial \mu \wedge \omega=0$, such that for every holomorphic vector field $U$ on $G$ we have

$$
0=i_{U}(\partial \mu \wedge \omega)=\left(i_{U} \partial \mu\right) \cdot \omega-\partial \mu \wedge\left(i_{U} \omega\right)
$$

or, equivalently,

$$
U(\mu) \cdot \omega=\partial \mu \wedge\left(i_{U} \omega\right)=\partial \mu \wedge \theta
$$

Now, definitions (2.1) and (3.1) yield the following result.

Proposition 3.2. A holomorphic function $\mu$ on $G$ is a holomorphic last multiplier for the holomorphic vector field $U$ if and only if

$$
U(\mu)+\mu \cdot \operatorname{div} U=0 .
$$

Remark 3.3. Relation (3.2) indicates that if $\nu$ is a holomorphic non-zero function on $G$ which satisfies the equation

$$
L_{U}(\nu):=U(\nu)=(\operatorname{div} U) \cdot \nu,
$$

then $1 / \nu$ is a holomorphic last multiplier for $U$ and the holomorphic function $\nu$ which satisfies (3.3) will be called an inverse holomorphic multiplier for $U$.

Proposition 3.4. Let $\mu$ be a holomorphic function on $G$. The set of holomorphic vector fields for which $\mu$ is a holomorphic last multiplier is a Lie subalgebra in the algebra of holomorphic vector fields on $G$.

Proof. The proof follows as in [6].

It is now interesting to search for a holomorphic last multiplier for a holomorphic vector field $U$ of divergence type, that is, $\mu=\operatorname{div} V$ for some holomorphic vector field $V$ on $G$. From (3.2),

$$
U(\operatorname{div} V)+\operatorname{div} V \cdot \operatorname{div} U=0 .
$$

Multiplying (3.4) by $\omega$ gives

$$
L_{U}(\operatorname{div} V) \cdot \omega+\operatorname{div} V \cdot L_{U} \omega=0
$$


or, equivalently,

$$
L_{U}(\operatorname{div} V \cdot \omega)=L_{U} L_{V} \omega=0 .
$$

Hence, we have

Proposition 3.5. If $V$ is a holomorphic vector field which satisfies $L_{U} L_{V} \omega=0$, then $\mu=\operatorname{div} V$ is a holomorphic last multiplier for the holomorphic vector field $U$.

The next step is to study holomorphic last multipliers for holomorphic gradient vector fields on the complex Lie group $G$ endowed with a holomorphic Riemannian metric (for instance $g$ or $h$ from Section 2). Such a metric $g$ defines a holomorphic metric volume form $\omega_{g}$ (see [10]), as a holomorphic $n$-form on $G$ such that

$$
\omega_{g}\left(U_{1}, \ldots, U_{n}\right)= \pm 1
$$

where $\left\{U_{i}\right\}, i=1, \ldots, n$, is an orthonormal holomorphic frame on $(G, g)$, that is, $g\left(U_{j}, U_{k}\right)=\delta_{j k}, j, k=1, \ldots, n$. As a complex manifold, if $(G, g)$ admits such a volume element, it admits precisely two of them.

If $f$ is a holomorphic function on $G, U=\operatorname{grad} f$ is the gradient vector field of $f$ defined in Section 2 and $\alpha$ is a holomorphic last multiplier for $U$, then relation (3.2) becomes

$$
g(\operatorname{grad} f, \operatorname{grad} \mu)+\mu \Delta f=0 .
$$

A straightforward computation in local complex coordinates on $G$ yields a similar identity to the case of holomorphic Riemannian manifolds, [6]:

$$
g(\operatorname{grad} f, \operatorname{grad} \mu)=\frac{1}{2}(\Delta(f \mu)-f \Delta \mu-\mu \Delta f) .
$$

Hence,

$$
\Delta(f \alpha)+\mu \Delta f=f \Delta \alpha
$$

which leads to the following result.

Proposition 3.6. Let $G$ be a complex Lie group endowed with a holomorphic metric $g$. If $f, \mu$ are holomorphic functions on $G$ such that $f$ is a holomorphic last multiplier for $\operatorname{grad} \mu$ and $\mu$ is a holomorphic last multiplier for $\operatorname{grad} f$, then $f \alpha$ is a holomorphic harmonic function on $G$.

Corollary 3.7. If $G$ is a complex Lie group endowed with a holomorphic metric $g$ and $f$ is a holomorphic function on $G$, then $\mu$ is a holomorphic last multiplier for $U=\operatorname{grad} \mu$ if and only if $\mu^{2}$ is a holomorphic harmonic function on $G$.

Corollary 3.8. If $G$ is a complex Lie group endowed with a holomorphic metric $g$ and $f$ is a holomorphic function on $G$, then $\mu^{2}$ is a holomorphic harmonic function on $G$ if and only if

$$
\mu \Delta \mu+g(\operatorname{grad} \mu, \operatorname{grad} \mu)=0 .
$$

Acknowledgement. The author would like to thank the anonymous referee for its remarks on the paper, which helped the author improve this note with more examples to illustrate the theoretical results presented here. This paper is supported by the Sectoral Operational Programme Human Resources Development (SOP HRD), ID134378 financed from the European Social Fund and by the Romanian Government. 


\section{References}

[1] Goldberg, S.I., Curvature and Homology, Revised Edition, Dover Publication, Inc. Mineola, New-York, 1998.

[2] Crâşmăreanu, M., Last multipliers theory on manifolds, Tensor, 66(2005), no. 1, 18-25.

[3] Crâşmăreanu, M., Last multipliers as autonomous solutions of the Liouville equation of transport, Houston J. Math., 34(2008), no. 2, 455-466.

[4] Crâşmăreanu, M., Last multipliers for multivectors with applications to Poisson geometry, Taiwanese J. Math., 13(2009), no. 5, 1623-1636.

[5] Crâşmăreanu, M., Last multipliers on weighted manifolds and the weighted Liouville equation, Politehn. Univ. Bucharest Sci. Bull. Ser. A Appl. Math. Phys., 77 (2015), no. 3 (in press).

[6] Crâşmăreanu, M., Ida, C., Popescu, P., Holomorphic last multipliers on complex manifolds, Preprint available to arXiv:submit/1295734 [math.DG] 4 July 2015.

[7] Ida, C., Ionescu, A., On a metric holomorphic connection in complex Lie groups, BSG Proceedings, 21(2014).

[8] Ionescu, A., On lifts of left-invariant holomorphic vector fields on complex Lie groups, Bulletin of Transilvania University, Series III, 7(2014), no. 2.

[9] Kobayashi, S., Nomizu, K., Foundations of Differential Geometry II, Wiley Interscience, New-York, 1969.

[10] LeBrun, C. R., H-Space with a cosmological constant, Proc. R. Soc. Lond. A, 380(1982), 171-185.

[11] Lee, D. H., The structure of complex Lie groups, Research Notes in Mathematics Series, Chapman and Hall/CRC, 232 pag.

[12] Toth, A., Varolin, D., Holomorphic diffeomorphisms of complex semisimple Lie groups, Inventiones Mathematicae, 139(2000), 351-369.

[13] Rund, H., Local differential-geometric structures on Lie groups, Tensor, N.S., 48(1998), 64-87.

[14] Wang, H.-C., Complex Parallelisable Manifolds, Proceedings of the American Math. Soc., 5(1954), no. 5, 71-776.

Alexandru Ionescu

"Transilvania" University

Faculty of Mathematics and Computer Sciences 50, Iuliu Maniu Street, 500050 Braşov, Romania e-mail: alexandru.codrin.ionescu@gmail.com 